\title{
The Association of Thyroid Hormones with Coronary Atherosclerotic Severity in Euthyroid Patients
}

Authors

Na Yu ${ }^{1}$, Linjie Wang ${ }^{1}$, Yong Zeng ${ }^{2}$, Yuxing Zhao ${ }^{1}$, Shi Chen ${ }^{1}$, Hui Pan ${ }^{1}$, Fengying Gong ${ }^{1}$, Huijuan Zhu ${ }^{1}$

Affiliations

1 Key Laboratory of Endocrinology of National Health Commission, Chinese Academy of Medical Science and Peking Union Medical College, Beijing, China

2 Department of Cardiology, Capital Medical University, Beijing, China

Key words

coronary artery disease, Gensini score, free triiodothyronine, FT3/FT4 ratio, euthyroid

received 03.08 .2021

accepted after revision $\quad 30.11 .2021$

Bibliography

Horm Metab Res 2022; 54: 12-19

DOI $10.1055 / a-1718-6283$

ISSN 0018-5043

C 2022. Thieme. All rights reserved.

Georg Thieme Verlag, Rüdigerstraße 14,

70469 Stuttgart, Germany

Correspondence

Huijuan Zhu

Key Laboratory of Endocrinology of National Health

Commission

Department of Endocrinology

State Key Laboratory of Complex Severe and Rare Diseases

Peking Union Medical College Hospital

Chinese Academy of Medical Science and Peking Union

Medical College

100730 Beijing

China

shengxin2004@163.com

Fengying Gong

Key Laboratory of Endocrinology of National Health

Commission

Department of Endocrinology

State Key Laboratory of Complex Severe and Rare Diseases

Peking Union Medical College Hospital

Chinese Academy of Medical Science and Peking Union

Medical College

100730 Beijing

China

Tel.: + 86/10/69151561

fygong@aliyun.com
丹 Supplementary material is available under

https://doi.org/10.1055/a-1718-6283.

\section{ABSTRACT}

The aim of the work was to explore the correlation between thyroid hormones and coronary atherosclerotic severity. This cross-sectional study included 340 euthyroid patients who underwent diagnostic coronary artery angiography (CAG). Gensini Score (GS) was applied to assess the severity of coronary atherosclerosis. Thyroid hormones and routine biochemical parameters were measured. The associations between thyroid hormones and coronary atherosclerosis severity were analyzed. Thyroid hormones levels or parameters were taken as both continuous variables and tertiles into analysis, and the lowest tertile was usually used as the reference $(O R=1)$ for medium and highest tertiles. Free triiodothyronine (FT3) level was associated with GS $\geq 22$ (Median GS) in Model I adjusted for age and sex [Continuous: $\mathrm{OR}=0.46,95 \% \mathrm{Cl}(0.23,0.92)$, $p=0.029$; Tertile 3: $O R=0.54,95 \% \mathrm{Cl}(0.30,0.97), p=0.038]$, and Model II adjusted for all known risk factors of coronary artery disease (CAD) [Continuous: $\mathrm{OR}=0.44,95 \% \mathrm{Cl}(0.20$, $0.95), p=0.036$; Tertile 3: $O R=0.49,95 \% \mathrm{Cl}(0.25,0.96)$, $\mathrm{p}=0.039]$. Subjects with highest tertile of FT3 to free thyroxine (FT4) ratio (FT3/FT4 ratio) appeared to have the remarkably decreased risk of CAD in both Non-adjusted Model [OR $=0.49$, $95 \% \mathrm{Cl}(0.24,0.98), \mathrm{p}=0.044]$ and Model I [OR $=0.45,95 \%$ $\mathrm{Cl}(0.22,0.93), \mathrm{p}=0.031]$. Higher FT3 level within normal range was independently and negatively associated with severity of coronary atherosclerosis. Besides, FT3/FT4 ratio was remarkably correlated with the prevalence of CAD in euthyroid population. 


\section{Introduction}

Despite increasing advances in the prevention and treatment of coronary artery disease (CAD), it has remained globally the single largest cause of mortality [1]. The inexorable progress of CAD, in spite of optimal guideline-based primary and secondary prevention, indicates the multifactorial etiology of the disease and the pressing need to explore other underlying mechanisms that may cause or exacerbate coronary atherosclerosis.

Thyroid hormones exert multiple effects on the cardiovascular system. Thyroid dysfunction leads to changes in endothelial function, systemic vascular resistance, blood pressure, cardiac contractility, cardiac output, and myocardial oxygen consumption [2]. The relationship between CAD and hyperthyroidism or hypothyroidism have been widely established for a long time $[3,4]$. Nevertheless, relatively less studies have focused on population with euthyroid status. For example, Steinberger et al. have reported free triiodothyronine (FT3) and free thyroxine (FT4) were positively associated with resting heart rate [5]. In addition, several observational studies demonstrated FT3 level was inversely associated with the presence of CAD or the severity of coronary atherosclerosis in euthyroid subjects [6-8], however, FT4, FT3 to FT4 ratio (FT3/FT4 ratio) and thyroid stimulating hormone (TSH) were not investigated in those studies. With regards to the relation between FT4 or TSH and CAD, the results of current research were conflicting [9-13]. Besides, the exploration of underlying confounders between thyroid hormones and CAD is insufficient.

Therefore, this study aimed to investigate the association between thyroid hormones levels or parameters (including FT3, FT4, FT3/FT4 ratio, and TSH) and the presence of CAD as well as severity of coronary atherosclerosis assessed by GS in euthyroid adults. We are also committed to explore potential confounding factor in the association.

\section{Subjects and Methods}

\section{Study Population}

There were overall 595 patients from North China with circulating thyroid function measurement results who underwent coronary artery angiography (CAG) at the Peking Union Medical College Hospital (PUMCH) between January 2013 and April 2016 for chest pain and precordial discomfort on basis of positive noninvasive test results (i. e., electrocardiogram suggestive of ischemia, suspicious coronary computed tomography angiography, positive exercise tolerance test, and so on). Out of the above participants, $147 \mathrm{pa}$ tients were excluded with prior history of percutaneous transluminal coronary intervention $(\mathrm{PCl})$ or coronary artery bypass grafting (CABG). Also 108 patients were excluded because of abnormal levels of thyroid hormones, medical history of hyperthyroidism or hypothyroidism. Finally, 340 euthyroid patients were studied, including 267 cases with CAD defined by diameter stenosis at $\geq 50 \%$ in at least one main branch of coronary artery, and 73 participants with all coronary artery stenosis $<50 \%$ as non-CAD ( $>$ Fig. $\mathbf{1}$ ).

\section{Ethics Approval}

Consent has been obtained from each patient after full explanation of the purpose and nature of all procedures. The protocol and the process of the study were approved by the Ethics Committee of Peking Union Medical College Hospital (No. S-K1580). The entire study was conducted in consistent with the Code of Ethics of the World Medical Association (Declaration of Helsinki) for experiments involving humans.

\section{Collection of blood samples and determination of variables}

Following an overnight fast for at least 8 hours, blood samples were collected from all patients and the total cholesterol (TC), low-density lipoprotein cholesterol (LDL-C), high-density lipoprotein cholesterol (HDL-C), triglycerides (TG), and hemoglobin A1c (HbA1c) were determined by conventional automated laboratory methods in the clinical laboratory of PUMCH.

The thyroid hormones including FT3, FT4, and TSH were tested by direct chemiluminescence method (ADVIA Centaur, Siemens, USA). The reference intervals of the laboratory in PUMCH were listed as follows: FT3, $1.80-4.10 \mathrm{pg} / \mathrm{ml}$; FT4, 0.81-1.89 ng/dl; TSH, $0.38-4.34 \mu \mathrm{lU} / \mathrm{ml}$. The subjects with circulating levels of thyroid hormones within the above reference range were taken as euthyroid patients. Patients with thyroid hormone levels out of the ref-

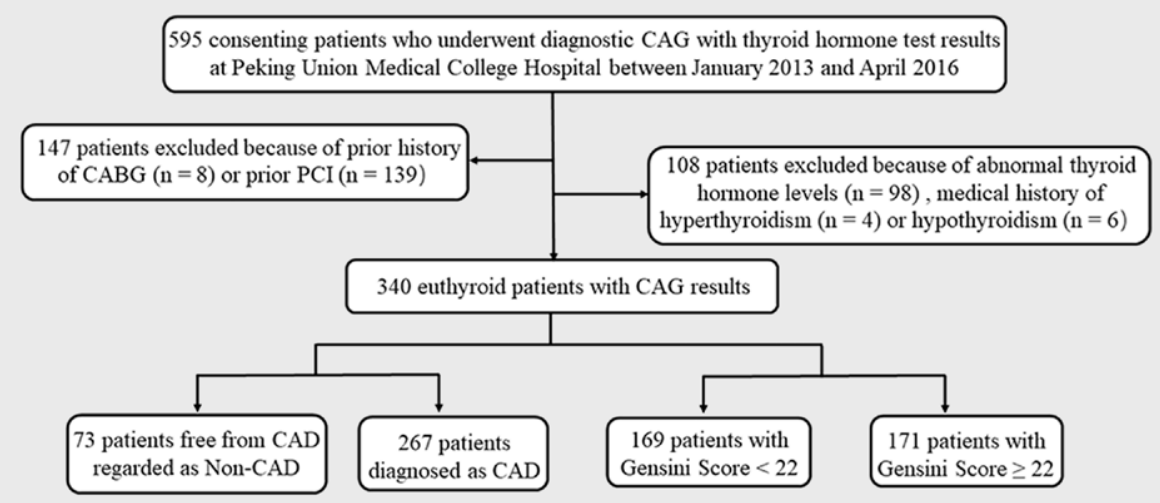

- Fig. 1 Flowchart of coronary atherosclerosis screening and quantification in patients. CAG: Coronary arteriography; CABG: Coronary artery bypass grafting; PCI: Percutaneous transluminal coronary intervention; CAD: Coronary artery disease. 
erence range (98 patients) and prior or current thyroid disease, including those taking thyroid medications (10 patients) were excluded ( $>$ Fig. 1).

\section{Assessment of coronary atherosclerotic severity}

Diagnostic CAG of each patient was performed with an angiography unit by an experienced cardiologist (Integris H; Philips Medical Systems, Amsterdam, the Netherlands). The imaging of coronary artery stenosis was completed from multiple projections. Based on the CAG results, CAD was diagnosed in cases for the presence of $\geq 50 \%$ stenosis in at least one main branch of coronary, while non-CAD were defined as $<50 \%$ stenosis in all coronary arteries. Furthermore, Gensini Score (GS) was calculated to quantify the coronary atherosclerotic severity by assigning a stenosis severity score to each coronary segment according to the degree of luminal narrowing and the detailed localization [14], the results of which were double checked by two separate researchers. The included participants were divided into the group of GS $\geq 22$ (171 patients) and the group of GS $<22$ ( 169 patients) with the median GS of them ( 22 scores) ( Fig. 1).

\section{Statistical analysis}

The continuous variables were expressed as mean \pm standard deviation or median with inter-quartile ranges if necessary. The categorical variables were represented as count and percentage. The Kruskal-Wallis test was performed for the comparison of continuous variables among tertile groups of FT3. And categorical variables were compared by Fisher exact test. Univariable and multivariate logistic regression analysis was applied to determine the independent association between thyroid hormone levels (including FT3, FT3/FT4 ratio, FT4 and TSH) and CAD or GS $\geq 22$. Additional stratified analysis and test for interaction of the association between FT3 and GS $\geq 22$ were conducted according to the following variables: age ( $<65$ years vs. $\geq 65$ years), sex (male vs. female), active smoking (yes vs. no) and body mass index (BMI) $\left(<24 \mathrm{~kg} / \mathrm{m}^{2}\right.$ vs. $\geq 24 \mathrm{~kg} / \mathrm{m}^{2}$ ). Multiple imputation, based on 5 replications and a chained equation approach method in the R MI procedure, was performed to account for missing data (including BMI, blood lipid levels and $\mathrm{HbA} 1 \mathrm{C}$ ) as sensitivity analysis. A two-tailed $\mathrm{p}<0.05$ was considered as statistically significant in all analyses. All the statistical analysis was performed using the Empower Stats (www.empowerstats.com, $X \& Y$ solutions, Inc. Boston, MA, USA) and R software version 3.6.1 (http://www.R-project.org). The forest plot was drawn with RStudio program, version 4.0.2 (http://www.R-project.org).

\section{Results}

\section{Study participants and baseline characteristics}

A total of 340 patients were included in the final analysis ( $\bullet$ Fig. 1). Participants were divided according to tertiles of FT3 levels and general characteristics of the study population, including demographics, clinical characteristics, laboratory indexes, and coronary atherosclerotic burden, are presented in $\triangleright$ Table $\mathbf{1}$. Compared to subjects with lower tertiles of FT3, those with the highest circulating FT3 levels showed a higher prevalence in women or smokers, and lower age and $\mathrm{HbA} 1 \mathrm{c}$ while higher FT4. BMI, history of chronic disease (such as hypertension, hyperlipidemia and diabetes), lipids profiles (including TC, HDL-C, LDL-C and TG), TSH, presence of CAD and GS did not differ statistically among three groups.

\section{Association between thyroid hormones and coronary atherosclerosis}

Multifactorial logistic regression analysis was performed to evaluate the relationship between thyroid hormones levels and atherosclerosis. FT3 level, taken as continuous variable or categorical variable in tertiles, was not statistically associated with the presence of CAD in non-adjusted Model and two adjusted models ( $p>0.05$ ). The lowest tertile of thyroid hormones levels or indexes was usually used as the reference $(O R=1)$ for medium and highest tertiles. The GS high than or equal to median value of included patients $(G S \geq 22)$ was used to represent severe coronary lesion. No significant association was observed between continuous FT3 and GS $\geq 22$ $[O R=0.66,95 \% \mathrm{Cl}(0.35,1.24), \mathrm{p}=0.197]$, neither was that between highest tertile of FT3 and GS $\geq 22$ compared to the lowest tertile $[\mathrm{OR}=0.75,95 \% \mathrm{Cl}(0.45,1.27), \mathrm{p}=0.285]$. However, the association between FT3 and GS $\geq 22$ became significant after the adjustment for several factors in two adjusted models (Model I and Model II). Specifically, in Model I, age and sex were adjusted for [Continuous FT3: $\mathrm{OR}=0.46,95 \% \mathrm{Cl}(0.23,0.92), \mathrm{p}=0.029$; Tertile 3 ofFT3: $\mathrm{OR}=0.54,95 \% \mathrm{Cl}(0.30,0.97), \mathrm{p}=0.038]$. Based on Model I, Model II was further adjusted for the following variables, including BMI, active smoking, hypertension, diabetes, $\mathrm{HbA} 1 \mathrm{c}$, LDL-C, HDL-C, and TG [Continuous FT3: OR $=0.44,95 \% \mathrm{Cl}(0.20,0.95)$, $p=0.036$; Tertile 3 of FT3: $O R=0.49,95 \% \mathrm{Cl}(0.25,0.96), p=0.039]$ ( $\triangleright$ Table 2). By contrast, subjects with the highest tertile of FT3/ FT4 ratio seemed to have the remarkably decreased risk of CAD in both non-adjusted Model $[\mathrm{OR}=0.49,95 \% \mathrm{Cl}(0.24,0.98), \mathrm{p}=0.044]$ and Model I [OR $=0.45,95 \% \mathrm{Cl}(0.22,0.93), \mathrm{p}=0.031]$. However, the reduced risk of CAD became insignificant in Model 2, the fully adjusted model. No significant association between FT3/FT4 ratio and GS $\geq 22$ was found ( $>$ Table 3 ).

Similar analysis was conducted to evaluate the relation between two other thyroid related hormones (including FT4 and TSH) and the presence of $C A D$ or $G S \geq 22$, afterwards, the correlations were not demonstrated $(p>0.05)$ (Tables S1, S2).

\section{Stratified analysis and test for interaction}

The stratified and interaction analysis were performed according to several potential confounders (including age, sex, smoking and $\mathrm{BMI}$ ) and the results are illustrated in $>$ Fig. 2. Each stratification was adjusted for all of variables (including age, sex, BMI, active smoking, hypertension, diabetes, HbA1c, LDL-C, HDL-C, and TG), except for the stratification factor itself. For example, circulating FT3 was noticeably associated with $\mathrm{GS} \geq 22$ in men [OR $=0.39,95 \%$ $\mathrm{Cl}(0.16,0.99), \mathrm{p}=0.048]$, smokers [OR $=0.34,95 \% \mathrm{Cl}(0.12,0.99)$, $\mathrm{p}=0.047]$ and subjects with age $<65$ years $[\mathrm{OR}=0.33,95 \% \mathrm{Cl}(0.13$, $0.84), p=0.019]$. No significant effect modifiers were found among these stratification factors ( $p$ for interaction $>0.05$ ). As is presented in Table S3, logistic regression analysis was conducted to estimate the association between the above three significant confounders (age, sex and active smoking) and FT3 levels or GS $\geq 22$. 
- Table 1 Baseline characteristics of the study participants according to tertiles of FT3.

\section{Tertiles of FT3}

\begin{tabular}{|c|c|c|c|c|c|}
\hline Characteristics & $\begin{array}{l}\text { All } \\
n=340\end{array}$ & $\begin{array}{l}T 1 \\
n=110\end{array}$ & $\begin{array}{l}T 2 \\
n=112\end{array}$ & $\begin{array}{l}\text { T3 } \\
n=118\end{array}$ & p-value \\
\hline \multicolumn{6}{|l|}{ Demographics } \\
\hline Age (years) & $59.6 \pm 11.7$ & $63.2 \pm 11.5$ & $61.2 \pm 10.1$ & $54.6 \pm 11.7$ & $<0.001$ \\
\hline Sex, male (n) & $231(67.9 \%)$ & $60(54.5 \%)$ & $32(28.6 \%)$ & $17(14.4 \%)$ & $<0.001$ \\
\hline $\operatorname{BMI}\left(\mathrm{kg} / \mathrm{m}^{2}\right)$ & $25.6 \pm 3.3$ & $25.1 \pm 3.6$ & $25.8 \pm 3.1$ & $25.9 \pm 3.2$ & 0.065 \\
\hline Active smoking (n) & $172(50.6 \%)$ & $43(39.1 \%)$ & $53(47.3 \%)$ & $76(64.4 \%)$ & $<0.001$ \\
\hline \multicolumn{6}{|l|}{ Clinical characteristics } \\
\hline Hypertension (n) & $233(68.5 \%)$ & $80(72.7 \%)$ & $75(67.0 \%)$ & $78(66.1 \%)$ & 0.510 \\
\hline Hyperlipidemia (n) & $209(61.5 \%)$ & $75(68.2 \%)$ & $61(54.5 \%)$ & $73(61.9 \%)$ & 0.110 \\
\hline Diabetes (n) & $126(37.1 \%)$ & $48(43.6 \%)$ & $43(38.4 \%)$ & $35(29.7 \%)$ & 0.087 \\
\hline \multicolumn{6}{|l|}{ Metabolic indicators } \\
\hline Total cholesterol (mmol/l) & $4.19 \pm 1.03$ & $4.19 \pm 1.08$ & $4.22 \pm 1.04$ & $4.18 \pm 0.99$ & 0.915 \\
\hline LDL cholesterol (mmol/l) & $2.52 \pm 0.91$ & $2.50 \pm 0.94$ & $2.50 \pm 0.92$ & $2.56 \pm 0.87$ & 0.675 \\
\hline HDL cholesterol (mmol/l) & $1.02 \pm 0.26$ & $1.03 \pm 0.27$ & $1.04 \pm 0.26$ & $0.99 \pm 0.26$ & 0.217 \\
\hline Triglyceride (mmol/l) & $1.64 \pm 1.15$ & $1.71 \pm 1.28$ & $1.60 \pm 1.16$ & $1.62 \pm 1.01$ & 0.940 \\
\hline $\mathrm{HbA1c}(\mathrm{mmol} / \mathrm{mol})$ & $46.30 \pm 14.78$ & $49.76 \pm 17.71$ & $45.94 \pm 13.51$ & $43.26 \pm 11.95$ & 0.009 \\
\hline \multicolumn{6}{|l|}{ Thyroid hormones } \\
\hline FT3 (pg/ml) & $2.99 \pm 0.34$ & $2.62 \pm 0.17$ & $2.97 \pm 0.09$ & $3.36 \pm 0.20$ & $<0.001$ \\
\hline FT4 (ng/dl) & $1.21 \pm 0.16$ & $1.14 \pm 0.15$ & $1.22 \pm 0.17$ & $1.25 \pm 1.14$ & $<0.001$ \\
\hline $\mathrm{TSH}(\mu \mathrm{lU} / \mathrm{ml})$ & $1.97 \pm 0.93$ & $2.00 \pm 0.94$ & $1.96 \pm 0.93$ & $1.95 \pm 0.92$ & 0.919 \\
\hline \multicolumn{6}{|c|}{ Coronary atherosclerotic burden } \\
\hline CAD (n) & $267(78.5 \%)$ & $88(80.0 \%)$ & 85 (75.9\%) & $94(79.7 \%)$ & 0.707 \\
\hline Gensini Score & $22[8,44]$ & $24[10,58]$ & $20[8,40]$ & $19[5,40]$ & 0.177 \\
\hline
\end{tabular}

Continuous data are presented as means \pm SD or medians (interquartile ranges), and categorical variables are presented as counts (\%). BMI: Body mass index; HbA1c: Hemoglobin A1c; LDL: Low-density lipoprotein; HDL: High-density lipoprotein; FT3: Free triiodothyronine; FT4: Free thyroxine; TSH: Thyroid-stimulating hormone; CAD: Coronary artery disease.

Age $<65$ years was positively related to FT3 levels compared with age $\geq 65$ years $[\beta=0.16,95 \% \mathrm{Cl}(0.09,0.24), \mathrm{p}<0.001]$, so were sex (male) $[\beta=0.24,95 \% \mathrm{Cl}(0.16,0.31), p<0.001]$ and active smoking $[\beta=0.13,95 \% \mathrm{Cl}(0.05,0.20), \mathrm{p}<0.001]$. Age $<65$ years was irrelevant to $G S \geq 22$ [OR $=0.97,95 \% \mathrm{Cl}(0.62,1.51)$, $\mathrm{p}=0.881$ ], however, sex (male) and active smoking were correlated with $G S \geq 22$ [Sex: $O R=1.91,95 \% \mathrm{Cl}(1.20,3.03), p=0.006$; Active smoking: $\mathrm{OR}=1.90,95 \% \mathrm{Cl}(1.23,2.92), \mathrm{p}=0.004]$.

\section{Sensitivity analysis}

As a cross-sectional study, missing data of adjusted variables was inevitable, including BMI $(n=35)$, blood lipid levels $(n=1)$ and $\operatorname{HbA1c}(n=24)$ in this study. Multiple imputation was used to evaluate the stability of critical results with missing data. As expected, both continuous FT3 levels and Tertile 3 of FT3 were stably associated with $\mathrm{GS} \geq 22$ after $\mathrm{MI}$ [Continuous: $\mathrm{OR}=0.52,95 \% \mathrm{Cl}(0.32$, $0.84), p=0.008$; Tertile 3: $O R=0.61,95 \% \mathrm{Cl}(0.40,0.93), p=0.021]$, which is presented in Table S4.

\section{Discussion}

\section{The major findings}

This cross-sectional study, performed in euthyroid Chinese patients who underwent the diagnostic CAG, explored the association between thyroid hormones levels or parameters (including FT3, FT4, FT3/FT4 ratio and TSH) and the presence of CAD or severe coronary atherosclerosis defined by $G S \geq 22$. The major finding was elevated FT3 level was significantly and inversely associated with higher $G S \geq 22$, independent of various traditional risk factors of CAD. This finding suggested low but clinically normal FT3 concentration might be potential risk factors for serious coronary atherosclerosis. An additional finding was the notable relation between FT3/FT4 ratio and the prevalence of CAD, following adjusted for age and sex. In comparison, neither FT4 nor TSH levels were established as significantly correlated factors for CAD or higher GS. 
- Table 2 Association between FT3 levels and severity of coronary atherosclerosis in multifactorial regression model.

\begin{tabular}{|c|c|c|c|c|c|c|c|}
\hline \multirow[b]{2}{*}{ Outcome } & & \multicolumn{2}{|c|}{ Non-adjusted Model } & \multicolumn{2}{|c|}{ Model I } & \multicolumn{2}{|c|}{ Model II } \\
\hline & & OR $(95 \% \mathrm{Cl})$ & p-Value & OR (95\% Cl) & p-Value & OR (95\% Cl) & p-Value \\
\hline \multirow[t]{5}{*}{ CAD } & Continuous FT3 (pg/ml) & $0.77(0.36,1.64)$ & 0.503 & $0.55(0.24,1.28)$ & 0.163 & $0.70(0.27,1.84)$ & 0.468 \\
\hline & Tertiles of FT3 & & & & & & \\
\hline & Tertile 1 & 1.00 & & 1.00 & & 1.00 & \\
\hline & Tertile 2 & $0.79(0.42,1.49)$ & 0.461 & $0.60(0.31,1.19)$ & 0.142 & $0.84(0.38,1.83)$ & 0.656 \\
\hline & Tertile 3 & $0.98(0.51,1.87)$ & 0.949 & $0.73(0.35,1.52)$ & 0.405 & $0.91(0.38,2.16)$ & 0.830 \\
\hline \multirow{5}{*}{$\begin{array}{l}\text { Gensini } \\
\text { Score } \geq 22\end{array}$} & Continuous FT3 (pg/ml) & $0.66(0.35,1.24)$ & 0.197 & $0.46(0.23,0.92)$ & 0.029 & $0.44(0.20,0.95)$ & 0.036 \\
\hline & Tertiles of FT3 & & & & & & \\
\hline & Tertile 1 & 1.00 & & 1.00 & & 1.00 & \\
\hline & Tertile 2 & $0.80(0.47,1.36)$ & 0.417 & $0.63(0.36,1.11)$ & 0.111 & $0.56(0.29,1.06)$ & 0.076 \\
\hline & Tertile 3 & $0.75(0.45,1.27)$ & 0.285 & $0.54(0.30,0.97)$ & 0.038 & $0.49(0.25,0.96)$ & 0.039 \\
\hline
\end{tabular}

Model I: Adjusted for age and sex. Model II: Adjusted for all of these variables, including age, sex, BMI, active smoking, hypertension, diabetes, LDL-C, HDL-C and TG.

- Table 3 Association between FT3/FT4 and severity of coronary atherosclerosis in multifactorial regression model.

\begin{tabular}{|c|c|c|c|c|c|c|c|}
\hline \multirow[b]{2}{*}{ Outcome } & & \multicolumn{2}{|c|}{ Non-adjusted model } & \multicolumn{2}{|c|}{ Model I } & \multicolumn{2}{|c|}{ Model II } \\
\hline & & OR (95\% Cl) & p-Value & OR (95\% Cl) & p-Value & OR (95\% Cl) & p-Value \\
\hline \multirow[t]{4}{*}{ CAD } & Tertiles of FT3/FT4 & & & & & & \\
\hline & Tertile 1 & 1.00 & & 1.00 & & 1.00 & \\
\hline & Tertile 2 & $0.79(0.39,1.63)$ & 0.531 & $0.70(0.33,1.46)$ & 0.338 & $0.91(0.37,2.26)$ & 0.841 \\
\hline & Tertile 3 & $0.49(0.24,0.98)$ & 0.044 & $0.45(0.22,0.93)$ & 0.031 & $0.48(0.20,1.11)$ & 0.087 \\
\hline \multirow{4}{*}{$\begin{array}{l}\text { Gensini } \\
\text { Score } \geq 22\end{array}$} & Tertiles of FT3/FT4 & & & & & & \\
\hline & Tertile 1 & 1.00 & & 1.00 & & 1.00 & \\
\hline & Tertile 2 & $1.00(0.58,1.72)$ & 0.996 & $0.91(0.53,1.59)$ & 0.111 & $1.13(0.59,2.16)$ & 0.722 \\
\hline & Tertile 3 & $0.80(0.46,1.39)$ & 0.427 & $0.76(0.43,1.33)$ & 0.336 & $0.79(0.41,1.51)$ & 0.477 \\
\hline
\end{tabular}

Model I: Adjusted for age and sex; Model II: Adjusted for all of these variables, including age, sex, BMI, active smoking, hypertension, diabetes, HbA1c, LDL-C, HDL-C, and TG.

\section{The interpretation of main findings}

We found FT3 was independently and negatively associated with severe coronary atherosclerosis. An observational study by Ertas et al. [8] proved lower FT3 levels within the normal range are an independent predictor of CAD and severe atherosclerosis of coronary (GS> 20) in 119 euthyroid Turkish patients. In another study [7] including 100 euthyroid Indian patients with stable angina and quantifying the severity of coronary stenosis with GS system, it was established that FT3 was negatively correlated with severe CAD. Homogeneously, Zhou et al. [6], who enrolled 4206 euthyroid Chinese patients, indicated that as normal FT3 levels declined, the presence and severity of CAD decreased in young population, but not in the old ones over 65 years old. Our results cohered with the study by
Zhou et al. [6] and we further investigated the FT3/FT4 ratio, FT4 and TSH simultaneously. We found firstly FT3/FT4 ratio was related to the prevalence of CAD, independent of age and sex. Whereas, FT3/FT4 ratio was not significantly correlated with the prevalence of coronary atherosclerosis in another study conducted in euthyroid population with Type 2 Diabetes [15]. This disparity was probably due to the heterogeneities between included participants, especially the different glucose metabolism status which is related to both thyroid hormones and artery atherosclerosis. Among a prospective cohort of 2106 euthyroid patients with triple vessel coronary artery disease [16], lower FT3/FT4 ratio was in connection with increased risks of long-term cardiac deaths as well as major adverse cardiac and cerebrovascular events (MACCEs), such 


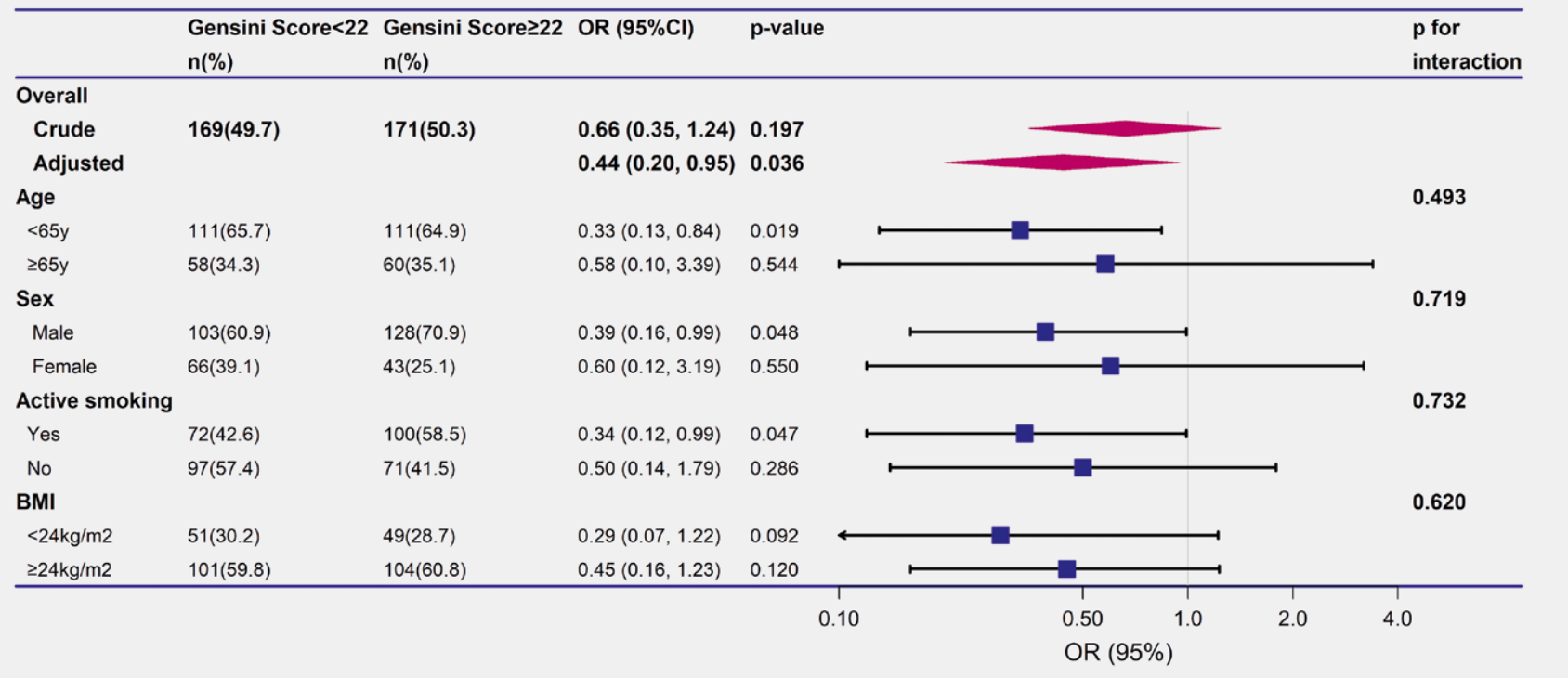

- Fig. 2 Stratified analyses of the association between FT3 and Gensini Score according to various baseline characteristics. The analysis was adjusted for all of these variables, including age, sex, BMI, active smoking, hypertension, diabetes, HbA1c, LDL-C, HDL-C, and TG, except for the specific stratification variable of that analysis.

as all-cause death, myocardial infarction, and stroke with the median follow-up for 5.3 years, suggesting the potential correlation between FT3/FT4 ratio with CAD.

Age, sex, and smoking were important factors, which were relevant to either FT3 or GS $\geq 22$ in this study. We found FT3 was independently associated with more severe coronary atherosclerosis in the subjects with age $<65$ years but not in the older ones, consistent with previous studies $[6,17]$. One of the possible reasons was depressed thyroid function, declined conversion of T4 to T3 and decreased sensitivity in organs to thyroid hormone with age [18]. Another possibility was the growing morbidity of glycemic and lipid metabolic problems, hypertension and other predictors of CAD in the aged, which may influence and interfere the impact of thyroid hormone on CAD. Similarly significant association between FT3 and GS $\geq 22$ in the males and smokers. It is not difficult to understand that sex and smoking are closely correlated with atherosclerosis. With regards to the correlation between them and FT3, there were different reasons. Circulating FT3 in males was higher than that in females throughout life except in the very young and very old, due to the potentiation effect of testosterone on the liver type 1 iodothyronine deiodinase (ID1), which catalyzes the deiodination of $\mathrm{T} 4$ followed by the production of biologically active T3 $[19,20]$. Cigarette smoking contributes to a rise of serum FT3 and FT4 induced by the activation of the sympathetic nervous system and abnormal immune profile in thyroid. All of those might be potential confounding factors between FT3 and CAD.

In our study, neither FT4 nor TSH levels were significantly correlated with prevalent CAD or higher GS over median value. Actually, FT4 and TSH within the normal reference range have been investigated in several studies and the conclusions have not been unified so far. For example, in a study [9] of 2045 consecutive adults no less than 30 years old, neither FT4 nor TSH were indicated to be associated with CAD. There were also hints that lower FT4 was in- versely related to coronary artery calcification and carotid intima media thickness (CIMT), the correlative risk factors of coronary atherosclerosis in euthyroid population [10,11]. Boggio et al. [12] revealed TSH levels in the upper limits of normal range was correlated with the less favorable cardiometabolic status and consequently with the increased risk of developing cardiovascular diseases, in euthyroid 744 Italian women. According to the research focused on older adults in the United States, increased TSH and decreased FT4 within normal limits were associated with reduced risk of multiple adverse events, including heart failure, atrial fibrillation, composite cardiovascular outcomes, and mortality [13]. Those studies led us to a novel comprehensive view that FT4 or TSH were mainly proved to be related to several risk factors or surrogate indicators of $C A D$, instead of CAD itself diagnosed with gold standard like CAG. However, the genesis and development of CAD might be influenced by multitudinous factors. Moreover, the discrepancy may derive from heterogeneities of participants in race, age and comorbidities to some extent. In this study, we explored the association between thyroid hormone and coronary atherosclerosis, which was quantified through CAG instead of surrogate indicators or risk factors in a Chinese population.

\section{The mechanism of effects of thyroid hormones on coronary atherosclerosis}

Thyroid hormones have been currently connected to endothelial function, blood pressure, lipids, insulin sensitivity, homocysteine, C-reactive protein, procoagulant state and so forth [2, 21-23], which may be mediating factors between FT3, the biologically active form of thyroid hormones, and coronary atherosclerosis. The production of nitric oxide (NO), which was revealed to mediate the rapid and direct vasodilatory effects of thyroid hormone at the level of isolated arteries [24,25], was declined in patients with overt or subclinical hypothyroidism and improved by thyroid hormone re- 
placement therapy [26-28]. Thyroxine (T4) tend to be converted to triiodothyronine (T3), the active form, under the action of deiodinase 2 (Dio2). Both long-term genomic and rapid non-genomic action of thyroid hormones occur upon binding T3 to thyroid hormone receptor- $\alpha$, causing the generation of NO ultimately [29]. Whereas, T4 only plays a role in non-genomic action by binding itself to integrin receptors before the NO is generated [29]. That might explain that FT3, the free form of T3, were more correlated with severe coronary atherosclerosis compared with FT4, the free form of T4. As a marker indicating the conversion rate from $\mathrm{T} 4$ to $\mathrm{T} 3, \mathrm{FT} 3 / \mathrm{FT} 4$ ratio was observed to negatively related to $C A D$, which could verify the speculation that $T 3$ exerts more protective effects in the occurrence and aggravation of CAD.

\section{The strengths and limitations of the study}

The strengths of this study are that all of our patients underwent standard CAG, the gold standard way of diagnosing CAD, to quantify the coronary atherosclerotic lesion, and that all of our patients were free from thyroid related history or drugs, which minimized underlying hybrid effects to a large degree. The limitations of study were unavoidable. First, this is a single-center study with limited sample size. Thus, our results need to be verified in multi-center study with larger sample size. Second, as a cross-sectional study, causal relationship could not be determined. Further prospective study is warranted. Last but not least, missing data of adjusted variables existed. However, we conducted sensitivity analysis through multiple imputation and confirmed the robustness of the results. Due to lack of data, the positive association between thyroid hormones and resting heart rate under euthyroid condition, which has been revealed by Steinberger et al. [5], was not explored further in this study.

\section{Conclusion}

In this study, we found even in euthyroid population, higher FT3 level may be significantly and inversely associated with severity of coronary atherosclerosis, independent of traditional risk factors of CAD. FT3/FT4 ratio may be remarkably correlated with the prevalence of $C A D$, following adjustment for age and sex. Our findings offered some enlightenments to importance of screening of thyroid function and appropriate levels of thyroid hormone. Further studies are warranted to verify the results and explore the underlying mechanisms.

\section{Conflicts of Interest}

The authors declare that they have no conflict of interest.

\section{References}

[1] Mendis SPP, Norrving B.(eds) Global atlas on CVD prevention and control.Geneva, Switzerland: World Health Organization; 2011Updated February 20, 2018. Available from: http://www.who.int/cardiovasculardiseases/publications/atlas_cvd/en/

[2] Razvi S, Jabbar A, Pingitore A et al. Thyroid hormones and cardiovascular function and diseases. J Am Coll Cardiol 2018; 71: 1781-1796
[3] Cooper DS, Biondi B. Subclinical thyroid disease. Lancet 2012; 379: 1142-1154

[4] Beyer C, Plank F, Friedrich G et al. Effects of hyperthyroidism on coronary artery disease: a computed tomography angiography study. Can J Cardiol 2017; 33: 1327-1334

[5] Steinberger E, Pilz S, Trummer C et al. Associations of thyroid hormones and resting heart rate in patients referred to coronary angiography. Horm Metab Res 2020; 52: 850-855

[6] Zhou BY, Guo YL, Wu NQ et al. Free triiodothyronine in relation to coronary severity at different ages: Gensini score assessment in 4206 euthyroid patients. J Geriatr Cardiol 2016; 13: 978-983

[7] Daswani R, Jayaprakash B, Shetty R et al. Association of thyroid function with severity of coronary artery disease in euthyroid patients. J Clin Diagn Res 2015; 9: OC10-OC13

[8] Ertaş F, Kaya H, Soydinç MS. Low serum free triiodothyronine levels are associated with the presence and severity of coronary artery disease in the euthyroid patients: an observational study. Anadolu Kardiyol Derg 2012; 12: 591-596

[9] Ling $\mathrm{Y}$, Jiang J, Gui M et al. Thyroid function, prevalent coronary heart disease, and severity of coronary atherosclerosis in patients undergoing coronary angiography. Int J Endocrinol 2015; 708272:

[10] Kim ES, Shin JA, Shin JY et al. Association between low serum free thyroxine concentrations and coronary artery calcification in healthy euthyroid subjects. Thyroid 2012; 22: 870-876

[11] Dullaart RP, de Vries R, Roozendaal C et al. Carotid artery intima media thickness is inversely related to serum free thyroxine in euthyroid subjects. Clin Endocrinol (Oxf) 2007; 67: 668-673

[12] Boggio A, Muzio F, Fiscella $M$ et al. Is thyroid-stimulating hormone within the normal reference range a risk factor for atherosclerosis in women? Intern Emerg Med 2014; 9: 51-57

[13] Cappola AR, Arnold AM, Wulczyn K et al. Thyroid function in the euthyroid range and adverse outcomes in older adults. J Clin Endocrinol Metab 2015; 100: 1088-1096

[14] Gensini GG. A more meaningful scoring system for determining the severity of coronary heart disease. Am J Cardiol 1983; 51: 606

[15] Wang L, Chen T, Yu J et al. Clinical associations of thyroid hormone levels with the risk of atherosclerosis in euthyroid type 2 diabetic patients in central China. Int J Endocrinol 2020; 2172781:

[16] Yuan D, Zhang C, Jia S et al. Predictive value of free triiodothyronine (FT3) to free thyroxine (FT4) ratio in long-term outcomes of euthyroid patients with three-vessel coronary artery disease. Nutr Metab Cardiovasc Dis 2020; 31: 579-586

[17] Hyland KA, Arnold AM, Lee JS et al. Persistent subclinical hypothyroidism and cardiovascular risk in the elderly: the cardiovascular health study. J Clin Endocrinol Metab 2013; 98: 533-540

[18] Rakov H, De Angelis M, Renko K et al. Aging is associated with low thyroid state and organ-specific sensitivity to thyroxine. Thyroid 2019; 29: $1723-1733$

[19] Miyashita K, Murakami M, Iriuchijima T et al. Regulation of rat liver type 1 iodothyronine deiodinase mRNA levels by testosterone. Mol Cell Endocrinol 1995; 115: 161-167

[20] Strich D, Karavani G, Edri S et al. FT3 is higher in males tah in females and decreases over the lifespan. Endocr Pract 2017; 23: 803-807

[21] Ichiki T. Thyroid hormone and vascular remodeling. J Atheroscler Thromb 2016; 23: 266-275

[22] Chadarevian R, Bruckert E, Leenhardt L et al. Components of the fibrinolytic system are differently altered in moderate and severe hypothyroidism. J Clin Endocrinol Metab 2001; 86: 732-737

[23] Klieverik LP, Janssen SF, van Riel A et al. Thyroid hormone modulates glucose production via a sympathetic pathway from the hypothalamic paraventricular nucleus to the liver. Proc Natl Acad Sci U S A 2009; 106: 5966-5971 
[24] Samuel S, Zhang K, Tang YD et al. Triiodothyronine potentiates vasorelaxation via PKG/VASP signaling in vascular smooth muscle cells. Cell Physiol Biochem 2017; 41: 1894-1904

[25] Zwaveling J, Pfaffendorf M, van Zwieten PA. The direct effects of thyroid hormones on rat mesenteric resistance arteries. Fundam Clin Pharmacol 1997; 11: 41-46

[26] Lekakis J, Papamichael C, Alevizaki M et al. Flow-mediated, endothelium-dependent vasodilation is impaired in subjects with hypothyroidism, borderline hypothyroidism, and high-normal serum thyrotropin (TSH) values. Thyroid 1997; 7: 411-414
[27] Taddei S, Caraccio N, Virdis A et al. Impaired endothelium-dependent vasodilatation in subclinical hypothyroidism: beneficial effect of levothyroxine therapy. J Clin Endocrinol Metab 2003; 88: 3731-3737

[28] Papaioannou GI, Lagasse M, Mather JF et al. Treating hypothyroidism improves endothelial function. Metabolism 2004; 53: 278-279

[29] Gaynullina DK, Schubert R, Tarasova OS. Changes in endothelial nitric oxide production in systemic vessels during early ontogenesis - a key mechanism for the perinatal adaptation of the circulatory system. Int J Mol Sci 2019; 20: 